\title{
Effect of Constant Magnetic Field on Convective Heat Transfer through Anisotropic River Beds
}

\author{
Julien Yovogan, Gérard Degan, Latif Fagbemi \\ Laboratoire d'Energétique et de Mécanique Appliquées (LEMA), Ecole Polytechnique d'Abomey-Calavi, Cotonou, Bénin \\ Email: yovojulien@yahoo.fr
}

How to cite this paper: Yovogan, J., Degan, G. and Fagbemi, L. (2018) Effect of Constant Magnetic Field on Convective Heat Transfer through Anisotropic River Beds. Journal of Crystallization Process and Technology, 8, 57-71.

https://doi.org/10.4236/jcpt.2018.82004

Received: January 13, 2018

Accepted: March 18, 2018

Published: March 21, 2018

Copyright () 2018 by authors and Scientific Research Publishing Inc. This work is licensed under the Creative Commons Attribution International License (CC BY 4.0).

http://creativecommons.org/licenses/by/4.0/

\begin{abstract}
An analytical investigation is conducted to study the effect of magnetic field on convection heat transfer through packed porous beds which consists of a horizontal fluid layer (river bed) and a porous zone with anisotropic permeability and underlined by a surface heated by a constant temperature $T_{1}$. The free surface of the fluid layer overlying the horizontal porous layer receives solar rays to length of day and is then considered heated isothermally at temperature $T_{2}$ such as $T_{1}<T_{2}$. Flow in porous medium is assumed to be governed by the generalized Brinkman-extended Darcy law and in the fluid layer by the Navier-Stokes model. The Beavers-Joseph condition is applied at the interface between the two layers. The influence of Hartmann number and hydrodynamic anisotropy on the convective phenomenon is investigated analytically. It is found that the magnetic field, the anisotropic permeability and the thickness of the porous lining, $\mathcal{E}$, have a strong influence of the geothermal convective flow and the heat transfer rate.
\end{abstract}

\section{Keywords}

Hartmann Number, Hydrodynamic Anisotropy, Convection Heat Transfer

\section{Introduction}

The first study concerning the effect of a magnetic field on the natural convection heat transfer in a rectangular porous cavity seems to be due to [1]. The vertical walls of the enclosure were maintained in isothermal and isoelectrical conditions. It was demonstrated that, for large Hartmann numbers, the electromagnetic magnetic force retards considerably the convective heat transfer. The influence of the Hartmann number on the Nusselt number was investigated numerically. The stability of a conducting fluid saturating a porous layer, in the 
presence of a uniform magnetic field, was investigated analytically by [2]. On the basis of the linear stability theory, the critical Rayleigh numbers for the onset of motion were obtained for various types of thermal and hydrodynamic boundary conditions. The case of a shallow cavity heated isothermally from the sides was considered by [3]. The effect of a magnetic field on the convective heat transfer was investigated analytically using matched asymptotic expansions. The results indicate that the retarding effect of the electromagnetic body Lorentz force decreases the strength of convection in the enclosure.

Several studies have been made on the effect of the magnetic field on the thermal convection through porous cavity. Our present research concerns the study of the thermal convection in a horizontal fluid-superposed porous layer and few studies have been made in this sense (see our literature magazine [4]). Like that, we consider the convective heat transfer through a parallel-plate horizontal system consisting of a homogeneous porous bed underlying a single-component fluid layer whose upper surface is free and isothermally heated. A magnetic field, $\boldsymbol{B}$, is applied perpendicularly to the long sides. The lower plate bounding the porous layer is impermeable and is maintained at a constant temperature. The porous medium is homogeneous and anisotropic in permeability whose principal axes are arbitrary oriented, as it is seen in nature and for many realistic applications. Beavers and Joseph boundary condition model is applied at the permeable bounding interface between the two layers [5]. On the basis of the generalized Brinkman-extended Darcy model, of Navier-Stokes equations and of energy equation which takes into account the viscous dissipation, the effects of magnetic field, of anisotropic parameters of the porous matrix and of the influence of the depth ratio on velocity and temperature fields and heat transfer rate are investigated in detail.

\section{Mathematical Formulation and Resolution}

The physical model illustrating the problem under different considerations is shown in Figure 1. The system of height, $h$, consists of a horizontal parallel-plate porous channel of thickness, $h_{m}$, underlying a fluid layer whose upper plate (free surface) is permeable and exposed to a constant temperature $T_{2}$. The lower impermeable plate lining the non-erodible porous layer is maintained at a constant temperature $T_{1}<T_{2}$.

The axial and transverse coordinates are respectively $x^{\prime}$ and $y^{\prime}$, the latter being measured vertically upwards from the lower impermeable wall. The porous medium is anisotropic in flow permeability, the permeabilities along the two principal axes of the porous matrix are denoted by $K_{1}$ and $K_{2}$. The anisotropy of the porous layer is characterized by the permeability ratio $K^{*}=K_{1} / K_{2}$ and the orientation angle $\varphi$, defined as the angle between the horizontal direction and the principal axis with the permeability $K_{2}$.

Thus, the flow regime is divided into two zones:

Zone 1 (fluid layer) from the free surface of the fluid to the surface of the 


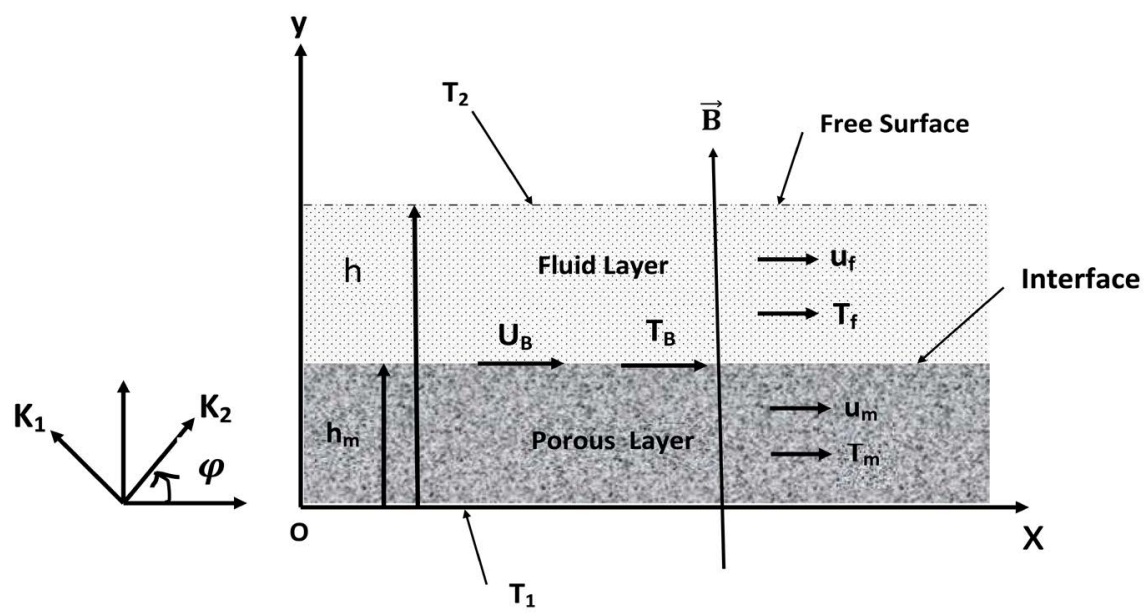

Figure 1. Physical model and coordinate system.

porous layer (called the nominal surface) and

Zone 2 (porous layer) from the nominal surface to the impermeable lower plate.

The porous bed is saturated with an incompressible viscous fluid that is in local thermodynamic equilibrium with the solid matrix.

The equations governing the conservation of mass, momentum, energy and electric charge transfer [4] [6] [7] can be written in each Zone as follows

Zone 1 (fluid layer):

Equation governing the conservation of mass

$$
\nabla \cdot V_{f}^{\prime}=0,
$$

Equation governing the conservation of momentum (Navier-Stokes model with the presence of magnetic and gravitational fields).

$$
\rho_{0}\left[\frac{\partial \boldsymbol{V}_{f}^{\prime}}{\partial t^{\prime}}+\left(\boldsymbol{V}_{f}^{\prime} \cdot \nabla\right) \boldsymbol{V}_{f}^{\prime}\right]=-\nabla \boldsymbol{p}_{f}+\rho_{f} \boldsymbol{g}+\mu_{e f f} \nabla^{2}\left(\boldsymbol{V}_{f}^{\prime}\right)+\boldsymbol{j}_{f} \wedge \boldsymbol{B},
$$

Equation governing the conservation of energy

$$
\frac{\partial T_{f}^{\prime}}{\partial t^{\prime}}+\left(\boldsymbol{V}_{f}^{\prime} \cdot \nabla\right) T_{f}^{\prime}=\alpha_{f} \nabla^{2}\left(T_{f}^{\prime}\right)+\frac{\mu}{\left(\rho C_{p}\right)_{f}} \Phi_{f},
$$

Equation governing the conservation of electric

$$
\nabla \cdot \boldsymbol{j}_{f}=0, \boldsymbol{j}_{f}=\sigma\left(-\nabla \varnothing_{f}+\boldsymbol{V}_{f}^{\prime} \wedge \boldsymbol{B}\right) .
$$

\section{Zone 2 (porous layer):}

Equation governing the conservation of mass

$$
\nabla \cdot V_{m}^{\prime}=0,
$$

Equation governing the conservation of momentum (Brinkman-extended Darcy law with the presence of magnetic and gravitational fields).

$$
\boldsymbol{V}_{m}^{\prime}=\frac{\overline{\bar{K}}}{\mu}\left(-\nabla p_{m}^{\prime}+\rho_{m} \boldsymbol{g}+\mu_{e f f} \nabla^{2}\left(\boldsymbol{V}_{m}^{\prime}\right)+\boldsymbol{j}_{m} \wedge \boldsymbol{B}\right),
$$


Equation governing the conservation of energy

$$
\left(\rho C_{p}\right)_{m} \frac{\partial T_{m}^{\prime}}{\partial t^{\prime}}+\left(\rho C_{p}\right)_{f} \nabla\left(V_{m}^{\prime} \cdot T_{m}^{\prime}\right)=k \nabla^{2}\left(T_{m}^{\prime}\right)+\mu \Phi_{m},
$$

Equation governing the conservation of electric

$$
\nabla \cdot \boldsymbol{j}_{m}=0, \boldsymbol{j}_{m}=\sigma\left(-\nabla \varnothing_{m}+\boldsymbol{V}_{m}^{\prime} \wedge \boldsymbol{B}\right) .
$$

In these equations, $\boldsymbol{V}$, denotes the velocity vector, $p^{\prime}$ the pressure and $T^{\prime}$ the temperature of the fluid, $\boldsymbol{g}$ the gravitational acceleration, $t^{\prime}$ the time, and $\left(\rho C_{p}\right)_{f},\left(\rho C_{p}\right)_{m}$ the heat capacity of the fluid and the saturated porous medium, respectively. The subscript " $P$ " denotes the fluid layer, " $m$ " the porous medium. Moreover, $\mu$ the dynamic viscosity, $\rho^{\prime}=\rho_{0}\left[1-\beta\left(T^{\prime}-T_{0}\right)\right]$ the density, $\beta$ the thermal-expansion coefficient, $T_{0}$ the constant reference Kelvin temperature, $\rho_{0}$ the density of the fluid at $T_{0}, C_{p}$ the specific heat of the fluid, $k$ the thermal conductivity and $\alpha=k /\left(\rho C_{p}\right)_{f}$ the thermal diffusivity. In Equation (3), $\boldsymbol{j}$ is the electric current density, $\sigma$ the electrical conductivity of the fluid, $\varnothing$ the electric potential and $-\nabla \varnothing$ the associated electric field. As discussed by [8], for a two dimensional situation Equation (4) and (8) for the electric potential reduces to $\nabla^{2} \varnothing=0$. The unique solution is $\nabla \varnothing=\mathbf{0}$. It follows that the electric field vanishes everywhere. The energy Equations (3) and (7) take into account the viscous dissipations $\Phi_{f}$ and $\Phi_{m}$ for the fluid layer and the porous medium respectively. It is important to mention here that the viscous dissipation $\Phi_{m}$ for the porous layer is the sum of viscous dissipation of the saturated fluid and the Darcy dissipation through the term $V_{m}^{\prime 2} / \overline{\bar{K}}$. In Equation (6), $\mu_{\text {eff }}$ denotes the apparent dynamic viscosity for Brinkman model and the symmetrical second-order permeability tensor $\overline{\bar{K}}$ is defined as

$$
\overline{\bar{K}}=\left[\begin{array}{ll}
K_{1} \sin ^{2} \varphi+K_{2} \cos ^{2} \varphi & \left(K_{2}-K_{1}\right) \sin \varphi \cos \varphi \\
\left(K_{2}-K_{1}\right) \sin \varphi \cos \varphi & K_{2} \sin ^{2} \varphi+K_{1} \cos ^{2} \varphi
\end{array}\right]
$$

Assuming that when the flow is fully developed in the system, the axial ( $x^{\prime}$-direction) velocity depends on the transverse coordinate $y^{\prime}$ (i.e., $u_{f}^{\prime}=u_{f}^{\prime}\left(y^{\prime}\right)$ for the fluid layer and $u_{m}^{\prime}=u_{m}^{\prime}\left(y^{\prime}\right)$ for the porous layer), and then from the continuity equation, the transverse velocity component must be zero (i.e., $v_{f}^{\prime}=0$ and $\left.v_{m}^{\prime}=0\right)$. The temperature is assumed to be a function of $y^{\prime}$. No assumptions are made with regard to the pressure variation (which, in fact, is found to be a function of $x^{\prime}$ and $y^{\prime}$ in Zone 2 [9]). So, governing Equations (1)-(8) may be reduced as

\section{Zone 1 (fluid layer):}

$$
\begin{gathered}
\frac{\mathrm{d} u_{f}^{\prime}}{\mathrm{d} x^{\prime}}=0 \\
-\frac{1}{\rho_{0}} \frac{\partial p_{f}^{\prime}}{\partial x^{\prime}}+v \frac{\mathrm{d}^{2} u_{f}^{\prime}}{\mathrm{d} y^{\prime 2}}-\frac{\sigma B^{2} u_{f}^{\prime}}{\rho_{0}}=0 \\
-\frac{1}{\rho_{0}} \frac{\partial p_{f}^{\prime}}{\partial y^{\prime}}=0
\end{gathered}
$$




$$
\alpha_{f} \frac{\mathrm{d}^{2} T_{f}^{\prime}}{\mathrm{d} y^{\prime 2}}+\frac{\mu}{\left(\rho C_{p}\right)_{f}}\left(\frac{\mathrm{d} u_{f}^{\prime}}{\mathrm{d} y^{\prime}}\right)^{2}=0 .
$$

Zone 2 (porous layer):

$$
\begin{gathered}
\frac{\mathrm{d} u_{m}^{\prime}}{\mathrm{d} x^{\prime}}=0 \\
b u_{m}^{\prime}=-\frac{K_{1}}{\mu} \frac{\partial p_{m}^{\prime}}{\partial x^{\prime}}+K_{1} \lambda \frac{\mathrm{d}^{2} u_{m}^{\prime}}{\mathrm{d} y^{\prime 2}}-\frac{K_{1} \sigma B^{2} u_{m}^{\prime}}{\mu}, \\
c u_{m}^{\prime}=\frac{K_{1}}{\mu} \frac{\partial p_{m}^{\prime}}{\partial y^{\prime}}, \\
k \frac{\mathrm{d}^{2} T_{m}^{\prime}}{\mathrm{d} y^{\prime 2}}=-\mu\left[\left(\frac{\mathrm{d} u_{m}^{\prime}}{\mathrm{d} y^{\prime}}\right)^{2}+\frac{b\left(u_{m}^{\prime}\right)^{2}}{K_{1}}\right] .
\end{gathered}
$$

where

$$
\left\{\begin{array}{l}
b=\sin ^{2} \varphi+K^{*} \cos ^{2} \varphi, \\
c=\left(1-K^{*}\right) \sin \varphi \cos \varphi .
\end{array}\right.
$$

And $\lambda=\mu_{\text {eff }} / \mu$ the relative viscosity for which the value in the present study is taken, as a first approximation, equal to unity (i.e., $\mu_{\text {eff }} \approx \mu$ ).

The appropriate boundary conditions prevailing on the lower impermeable boundary and the upper free surface and at the interface of the two layers $(y=$ $h_{m}$ ) of the channel are [4]

$$
\begin{gathered}
y^{\prime}=0: u_{m}^{\prime}=0, T_{m}^{\prime}=T_{1}, \\
y^{\prime}=h: \frac{\mathrm{d} u_{f}^{\prime}}{\mathrm{d} y^{\prime}}=0, T_{f}^{\prime}=T_{2}, \\
y^{\prime}=h_{m}: u_{f}^{\prime}=u_{m}^{\prime}=u_{B} / \frac{\mathrm{d} u_{f}^{\prime}}{\mathrm{d} y^{\prime}}=\frac{\beta_{1}}{\sqrt{K_{1}}}\left(u_{B}-\left.u_{m}^{\prime}\right|_{y=h_{m}-\sqrt{K_{1}}}\right), \\
y^{\prime}=h_{m}: T_{f}^{\prime}=T_{m}^{\prime}=T_{B} / \frac{\mathrm{d} T_{f}^{\prime}}{\mathrm{d} y^{\prime}}=\frac{\beta_{2}}{\sqrt{K_{1}}}\left(T_{B}-\left.T_{m}^{\prime}\right|_{y=h_{m}-\sqrt{K_{1}}}\right) .
\end{gathered}
$$

where $u_{B}$ is the slip velocity at the nominal surface (interface) which changes to the constant Darcy velocity through the porous, the existence of the temperature slip layer whose thickness given by Beavers and Joseph is of order $\sqrt{K_{1}}$. Indeed, this thickness subsequently has been shown to be equal to $\sqrt{K_{1}}$ [10] [11]. According to these authors, the parameter $\beta_{1}$ (and $\beta_{2}$ ) denotes a constant depending on the material property of the porous medium, which have can be determined only experimentally. The slip temperature $T_{B}$ at the nominal surface changes to the constant ambient temperature $T_{0}$ through the porous layer whose thickness considered to be the same as that of the velocity slip layer namely $\sqrt{K_{1}}$. So, the existence of the temperature slip layer depends on that of the velocity slip layer and in view of this, $T_{0}$ is identified with $T_{m}^{\prime}$ at a distance $\sqrt{K_{1}}$ below the nominal. 
When the following variables are introduced

$$
\left.\begin{array}{l}
x=x^{\prime} /(h R e), y=y^{\prime} / h, \\
\left(u_{f}, u_{m}\right)=\left(u_{f}^{\prime}, u_{m}^{\prime}\right) / \bar{u},\left(T_{f}, T_{m}\right)=\left[\left(T_{f}^{\prime}, T_{m}^{\prime}\right)-T_{1}\right] / \Delta T, \\
\Delta T=T_{2}-T_{1},\left(P_{f}, P_{m}\right)=\left(p_{f}^{\prime}, p_{m}^{\prime}\right) /\left(\rho \bar{u}^{2}\right) .
\end{array}\right\}
$$

the governing Equations (10)-(13) corresponding to the fluid layer may be written in non-dimensional form as

$$
\begin{aligned}
& \frac{\mathrm{d}^{2} u_{f}}{\mathrm{~d} y^{2}}-H a^{2} u_{f}=\frac{\mathrm{d} P_{f}}{\mathrm{~d} x}, \\
& \frac{\mathrm{d}^{2} T_{f}}{\mathrm{~d} y^{2}}=-\operatorname{PrEc}\left(\frac{\mathrm{d} u_{f}}{d y}\right)^{2} .
\end{aligned}
$$

and the governing Equations (14)-(17) corresponding to the anisotropic porous layer may be written in non-dimensional form as

$$
\begin{gathered}
\frac{\mathrm{d}^{2} u_{m}}{\mathrm{~d} y^{2}}-\frac{b+H a_{m}^{2}}{D a} u_{m}=\frac{\partial P_{m}}{\partial x}, \\
\frac{\partial P_{m}}{\partial y}=\frac{c}{\operatorname{ReDa}} u_{m}, \\
\frac{\mathrm{d}^{2} T_{m}}{\mathrm{~d} y^{2}}=-\operatorname{PrEc}\left[\left(\frac{\mathrm{d} u_{m}}{\mathrm{~d} y}\right)^{2}+\gamma^{2}\left(u_{m}\right)^{2}\right] .
\end{gathered}
$$

where

$$
\gamma^{2}=\frac{b}{D a}
$$

In the above equations, $\operatorname{Pr}=\mu C_{p} / k$ is the Prandtl number, $E c=\bar{u}^{2} /\left(C_{p} \Delta T\right)$ the Eckert number, $R e=\bar{u} h / v$ the Reynolds number, $D a=K_{1} / h^{2}$ the Darcy number, $H a=B \cdot h \sqrt{\sigma / \mu}$ the Hartmann number for the fluid layer and $H a_{m}=B \sqrt{K_{1} \sigma / \mu}$ the Hartmann number for the anisotropic porous layer.

The boundary conditions associated with the non-dimensional Equations (19)-(22) are [4]

$$
\begin{gathered}
y=0: u_{m}=0, T_{m}=0, \\
y=1: \frac{\mathrm{d} u_{f}}{\mathrm{~d} y}=0, T_{f}=1, \\
y=\varepsilon: u_{f}=u_{m}=u_{B} / \frac{\mathrm{d} u_{f}}{\mathrm{~d} y}=\frac{\beta_{1}}{\sqrt{D a}}\left(u_{B}-\left.u_{m}\right|_{y=\varepsilon-\sqrt{D a}}\right), \\
y=\varepsilon: T_{f}=T_{m}=T_{B} / \frac{\mathrm{d} T_{f}}{\mathrm{~d} y}=\frac{\beta_{2}}{\sqrt{K_{1}}}\left(T_{B}-\left.T_{m}\right|_{y=\varepsilon-\sqrt{D a}}\right) .
\end{gathered}
$$

where $\varepsilon=h_{m} / h$ is the thickness ratio defining as the ratio of saturated porous layer thickness to packed porous beds thickness (which consists of a horizontal fluid layer (river bed) and a porous zone). 
Using the hydrodynamic conditions (30) and (32), the resolution of Equation (24) yields the velocity distribution in Zone 1 (for the fluid layer) expressed as

$$
u_{f}(y)=A_{1} \mathrm{e}^{H a \cdot y}+A_{2} \mathrm{e}^{-H a \cdot y}-\frac{Q}{H a^{2}}
$$

where $Q=\mathrm{d} P_{f} / \mathrm{d} x$.

Taking into account Equation (34) and considering the boundary conditions for $T_{f}$, Equation (25) can be integrated to give the following fully developed temperature profile in fluid Zone:

$$
T_{f}(y)=-P r E C\left[\frac{A_{1}^{2}}{4} \mathrm{e}^{2 \cdot H a \cdot y}+\frac{A_{2}^{2}}{4} \mathrm{e}^{-2 \cdot H a \cdot y}-A_{1} A_{2} H a^{2} y\right]+A_{3} \cdot y+A_{4},
$$

where

$$
\left.\begin{array}{l}
A_{1}=\frac{U_{B}+Q / H a^{2}}{\left[\mathrm{e}^{H a \cdot \varepsilon}+\mathrm{e}^{H a(2-\varepsilon)}\right]}, \\
A_{2}=A_{1} \mathrm{e}^{2 \cdot H a}, \\
A_{3}=\frac{T_{B}-1}{\varepsilon-1}+\frac{\operatorname{PrEC}}{4(\varepsilon-1)}\left[\left(\mathrm{e}^{2 \cdot H a \cdot \varepsilon}+\mathrm{e}^{2 \cdot H a(2-\varepsilon)}\right)+2\left[2 H a^{2}\left(1-\varepsilon^{2}\right)-1\right] \mathrm{e}^{2 \cdot H a}\right] A_{1}^{2}, \\
A_{4}=\frac{\varepsilon-T_{B}}{\varepsilon-1}+\frac{\operatorname{PrEC}}{4(\varepsilon-1)}\left[2 \cdot \varepsilon \cdot \mathrm{e}^{2 \cdot H a}\left(1-2 H a^{2}(1-\varepsilon)\right)-\left(\mathrm{e}^{2 \cdot H a \cdot \varepsilon}+\mathrm{e}^{2 \cdot H a(2-\varepsilon)}\right)\right] A_{1}^{2} \cdot
\end{array}\right\}
$$

where $u_{B}$ is the velocity profile at the interface that must be determined by making use of the condition (32) in which we have to know first the velocity $u_{m}$ for the porous layer. It is seen that $T_{f}$ depends on $T_{B}$ that has to be calculated from the boundary condition (33) for which the temperature profile $T_{m}$ (in Zone 2) should be expressed.

Now, the velocity and temperature profiles $u_{m}$ and $T_{m}$ for the porous layer will be determined.

As $H a_{m}^{2}=\mathrm{Da} \cdot H a^{2}$, Equation(26) may be written as

$$
\frac{\mathrm{d}^{2} u_{m}}{\mathrm{~d} y^{2}}-\xi^{2} u_{m}=\frac{\partial P_{m}}{\partial x}
$$

where $\xi^{2}=\gamma^{2}+H a^{2}$.

Eliminating the pressure from Equation (27) and (37) in the usual way, one may have

$$
\frac{\mathrm{d}^{3} u_{m}}{\mathrm{~d} y^{3}}-\xi^{2} \frac{\mathrm{d} u_{m}}{d y}=0
$$

It is clear that the bulk mean velocity $\vec{u}$ (in Zone 2 ) defined as $\vec{u}=\left(1 /\left[\rho^{\prime}\left(h_{m} \times 1\right)\right]\right) \int_{0}^{h_{m}} \rho^{\prime} u_{m}^{\prime} \mathrm{d} y^{\prime}$ is calculated in dimensionless terms by

$$
\bar{u}=\int_{0}^{\varepsilon} u_{m} \mathrm{~d} y
$$

which equals to $\varepsilon$. 
Making use of Equation (39) and the hydrodynamic conditions (31) and (32), the resolution of Equation (38) yields the velocity distribution (for the porous layer) expressed as

$$
u_{m}(y)=\frac{1}{\xi}\left(B_{1} \mathrm{e}^{\xi y}-B_{2} \mathrm{e}^{-\xi y}\right)+B_{3}
$$

where

$$
\left.\begin{array}{l}
B_{1}=\frac{\xi^{2} \varepsilon\left(1-\mathrm{e}^{-\xi \varepsilon}\right)-u_{B}\left(\mathrm{e}^{-\xi \varepsilon}+\xi \varepsilon-1\right) \xi}{4 \cdot \cosh (\xi \varepsilon)-2 \cdot \xi \cdot \varepsilon \cdot \sinh (\xi \varepsilon)-4} \\
B_{2}=\frac{B_{1}\left(1-\mathrm{e}^{\xi \varepsilon}\right)+\xi u_{B}}{1-\mathrm{e}^{-\xi \varepsilon}} \\
B_{3}=\frac{B_{2}-B_{1}}{\xi}
\end{array}\right\}
$$

By substituting Equation (40) into Equation (28) and using the thermal boundary conditions (32) and (33), the temperature profile for the porous medium is obtained as

$$
\begin{aligned}
T_{m}(y)= & -\operatorname{PrEc}\left[\frac{1}{4 \cdot \xi^{2}}\left(1+\frac{\gamma^{2}}{\xi^{2}}\right)\left(B_{1}^{2} \mathrm{e}^{2 \xi y}+B_{2}^{2} \mathrm{e}^{-2 \xi y}\right)\right. \\
& +\frac{2 B_{1} \gamma^{2}}{\xi^{3}}\left(B_{1} \mathrm{e}^{\xi y}-B_{2} \mathrm{e}^{-\xi y}\right) \\
& \left.+\left(2\left(1-\frac{\gamma^{2}}{\xi^{2}}\right) B_{1} B_{2}+\gamma^{2} B_{3}^{2}\right) \frac{y^{2}}{2}\right]+B_{4} \cdot y+B_{5} .
\end{aligned}
$$

where

$$
\begin{aligned}
B_{4}= & \frac{T_{B}-B_{5}}{\varepsilon}+\frac{\operatorname{PrEC}}{\varepsilon}\left[\frac{1}{4 \cdot \xi^{2}}\left(1+\frac{\gamma^{2}}{\xi^{2}}\right)\left(B_{1}^{2} \mathrm{e}^{2 \xi \varepsilon}+B_{2}^{2} \mathrm{e}^{-2 \xi \varepsilon}\right)\right. \\
& \left.+\frac{2 B_{3} \gamma^{2}}{\xi^{3}}\left(B_{1} \mathrm{e}^{\xi \varepsilon}-B_{2} \mathrm{e}^{-\xi \varepsilon}\right)+\left(2\left(1-\frac{\gamma^{2}}{\xi^{2}}\right) B_{1} B_{2}+\gamma^{2} B_{3}^{2}\right) \frac{\varepsilon^{2}}{2}\right], \\
B_{5}= & \operatorname{PrEc}\left[\frac{1}{4 \cdot \xi^{2}}\left(1+\frac{\gamma^{2}}{\xi^{2}}\right)\left(B_{1}^{2}+B_{2}^{2}\right)+\frac{2 B_{3} \gamma^{2}}{\xi^{3}}\left(B_{1}-B_{2}\right)\right]
\end{aligned}
$$

It is noticed that both the velocity profiles $u_{f}$ and $u_{m}$ depend on $u_{B}$. the same holds good for both the temperature profiles $T_{f}$ and $T_{m}$ which depend on $T_{B}$. The profiles $u_{B}$ and $T_{B}$ at the interface are determined by using boundary conditions (32) and (33) respectively. After manipulations, one can obtain

$$
u_{B}=\frac{a_{5} \cdot H a \cdot \beta_{1}-Q \cdot a_{7} \cdot \sqrt{D a}}{H a\left(H a \cdot a_{7} \sqrt{D a}-\beta_{1}\left(1+a_{6}\right)\right)}
$$

where 


$$
\begin{aligned}
& a_{5}=a_{1} \frac{1-\mathrm{e}^{\xi(\varepsilon-\sqrt{D a})}}{\varepsilon}-a_{3} \frac{1-\mathrm{e}^{-\xi(\varepsilon-\sqrt{D a})}}{\varepsilon} \\
& a_{6}=a_{2} \frac{1-\mathrm{e}^{\xi(\varepsilon-\sqrt{D a})}}{\varepsilon}-a_{4} \frac{1-\mathrm{e}^{-\xi(\varepsilon-\sqrt{D a})}}{\varepsilon} \\
& a_{7}=\frac{\mathrm{e}^{H a \cdot \varepsilon}-\mathrm{e}^{H a(2-\varepsilon)}}{\mathrm{e}^{H a \cdot \varepsilon}+\mathrm{e}^{H a(2-\varepsilon)}} \\
& a_{1}=\frac{\xi^{2} \varepsilon\left(1-\mathrm{e}^{-\xi \varepsilon}\right)}{a_{0}} \\
& a_{0}=4 \cdot \cosh (\xi \varepsilon)-2 \cdot \xi \cdot \varepsilon \cdot \sinh (\xi \varepsilon)-4 \\
& a_{2}=\frac{1-\xi \varepsilon-\mathrm{e}^{-\xi \varepsilon}}{a_{0}} \\
& a_{3}=\frac{a_{1}\left(1-\mathrm{e}^{\xi \varepsilon}\right)}{1-\mathrm{e}^{-\xi \varepsilon}} \\
& a_{4}=\frac{\xi+a_{2}\left(1-\mathrm{e}^{\xi \varepsilon}\right)}{1-\mathrm{e}^{-\xi \varepsilon}}
\end{aligned}
$$

Similarly, after development and reduction of algebraic terms, the characteristic temperature distribution $T_{B}$ at the interface is calculated by

$$
T_{B}=\frac{\left[b_{0} \sqrt{D a}+\left(b_{1}+b_{2}\right) \beta_{2}\right] \varepsilon(1-\varepsilon)}{\beta_{2} \cdot \varepsilon(1-\varepsilon)+(\sqrt{D a}-\varepsilon) \beta_{2}(1-\varepsilon)+\sqrt{D a}}
$$

where

$$
\begin{aligned}
& b_{0}=\frac{\operatorname{PrEc}}{4(\varepsilon-1)}\left[(1-2 \cdot H a(\varepsilon-1)) \mathrm{e}^{2 \cdot H a \cdot \varepsilon}+(1+2 \cdot H a(\varepsilon-1)) \mathrm{e}^{2 \cdot H a(2-\varepsilon)}+b_{3}\right] A_{1}^{2}+\frac{1}{\varepsilon-1} \\
& b_{1}=-\operatorname{PrEc}\left[\frac{1}{4 \cdot \xi^{2}}\left(1+\frac{\gamma^{2}}{\xi^{2}}\right)\left(B_{1}^{2} \mathrm{e}^{2 \xi(\varepsilon-\sqrt{D a})}+B_{2}^{2} \mathrm{e}^{-2 \xi(\varepsilon-\sqrt{D a})}\right)+b_{3}\right] \\
& b_{2}=\frac{\varepsilon-\sqrt{D a}}{\varepsilon}\left[\operatorname{PrEc}\left(\frac{1}{4 \cdot \xi^{2}}\left(1+\frac{\gamma^{2}}{\xi^{2}}\right)\left(B_{1}^{2} \mathrm{e}^{2 \xi \varepsilon}+B_{2}^{2} \mathrm{e}^{-2 \xi \varepsilon}\right)+b_{5}\right)-B_{5}\right] \\
& \left.b_{3}=\left[8 \cdot \varepsilon H a^{2}(\varepsilon-1)+2 \cdot\left(2 \cdot H a^{2}\left[1-\varepsilon^{2}\right]-1\right)\right] \mathrm{e}^{2 \cdot H a}\right] \\
& b_{4}=\frac{2 B_{3} \gamma^{2}}{\xi^{3}}\left(B_{1} \mathrm{e}^{\xi(\varepsilon-\sqrt{D a})}-B_{2} \mathrm{e}^{-\xi(\varepsilon-\sqrt{D a})}\right)+\left(2\left(1-\frac{\gamma^{2}}{\xi^{2}}\right) B_{1} B_{2}+\gamma^{2} B_{3}^{2}\right)\left(\frac{\varepsilon-\sqrt{D a})^{2}}{2}\right. \\
& b_{5}=\frac{2 B_{3} \gamma^{2}}{\xi^{3}}\left(B_{1} \mathrm{e}^{\xi \varepsilon}-B_{2} \mathrm{e}^{-\xi \varepsilon}\right)+\left(2\left(1-\frac{\gamma^{2}}{\xi^{2}}\right) B_{1} B_{2}+\gamma^{2} B_{3}^{2}\right) \frac{\varepsilon^{2}}{2}
\end{aligned}
$$

The heat transfer rates through the upper free surface and the lower wall bounding the porous layer underlining the fluid zone are expressed by the Nusselt numbers given in dimensionless terms respectively by

$$
N u_{u}=\left.\frac{\mathrm{d} T_{f}}{\mathrm{~d} y}\right|_{y=1}=2 \cdot \operatorname{PrEc} \cdot H a^{2} A_{1}^{2} \mathrm{e}^{2 \cdot H a}+A_{3}
$$


and

$$
N u_{\ell}=\left.\frac{\mathrm{d} T_{m}}{\mathrm{~d} y}\right|_{y=0}=-\operatorname{PrEc}\left(1-\frac{\gamma^{2}}{\xi^{2}}\right) \frac{B_{1}-B_{2}}{2 \cdot \xi}+\frac{2 B_{3} \gamma^{2}}{\xi^{3}}\left(B_{1}+B_{2}\right)+B_{4}
$$

Consequently, one can deduce that the mean heat transfer rate $N u_{\text {moy }}$ through the superposed layers is calculated by the following expression

\section{Results and Discussion}

\subsection{Horizontal Velocity Distribution and Temperature Distribution for the Entire System Width}

Figure 2 illustrates the analytical results obtained for the Horizontal velocity distribution for the entire system width when $D a=0.05, Q=-2, \beta_{1}=0.1, \mathcal{E}=$ $0.2, K^{*}=0.1, \varphi=0^{\circ}$ and various values of $\mathrm{Ha}$. From this figure it is clearly seen that the velocity distribution decreases as the Hartmann number increases. For $K^{*}=0.1$ (When the permeability in the horizontal direction, $K_{2}$, is higher than the permeability in the vertical direction, $K_{1}$.) the convective flow is high when $\mathrm{Ha}=0.8$ (and for the lowest values of $\mathrm{Ha}$ ) and is reduced when $\mathrm{Ha}=5$ (and for the highest values of $\mathrm{Ha}$ ). It is noticed that the velocity fields have the same behavior as described previously [4].

Figure 3 illustrates the analytical results obtained for Temperature distribution for the entire system width when $K^{*}=0.1, \beta_{1}=\beta_{2}=0.1, D a=005, Q=-2, \mathcal{E}$ $=0.4, \operatorname{Pr}=7, E c=0.05, \varphi=0^{\circ}$. It is noticed that the temperature fields have the same behavior as described previously for the velocity distribution, revealing that the effect of varying convection in the two superposed layers depends strongly on the magnetic field, i.e., Ha.

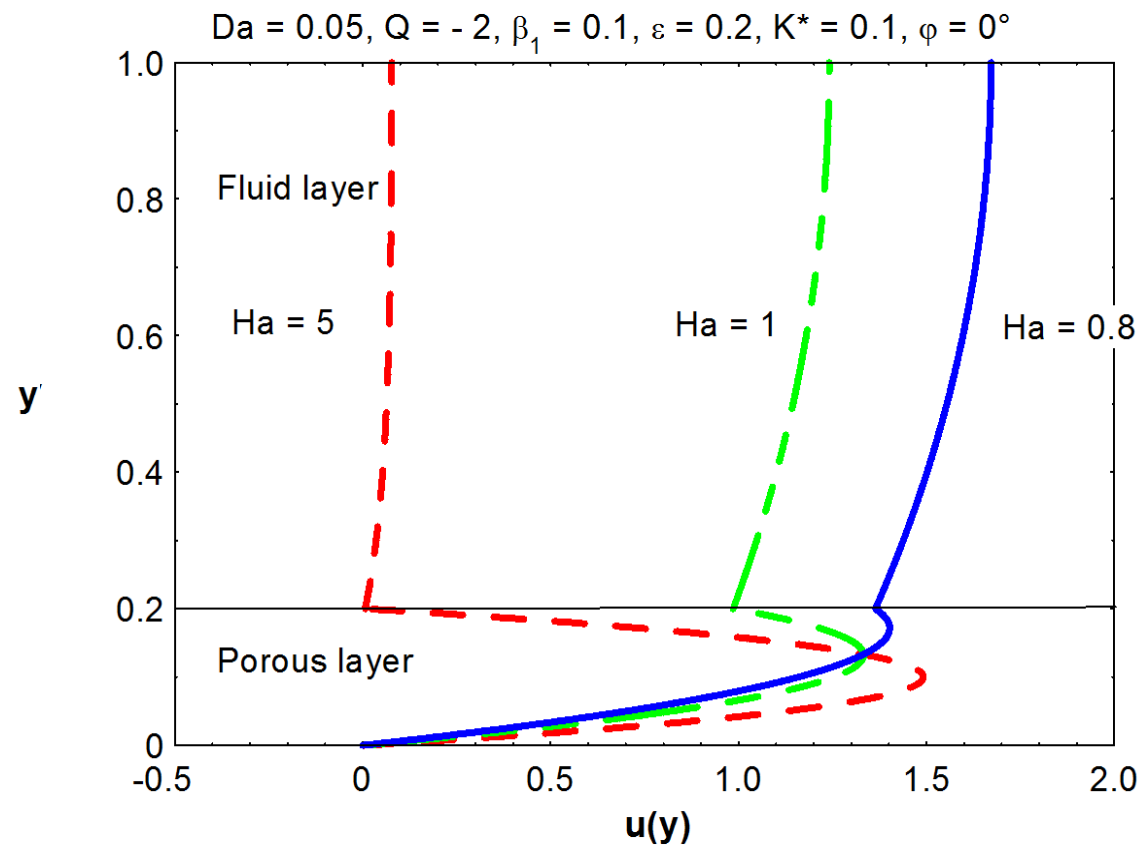

Figure 2. Horizontal velocity distribution for the entire system width. 


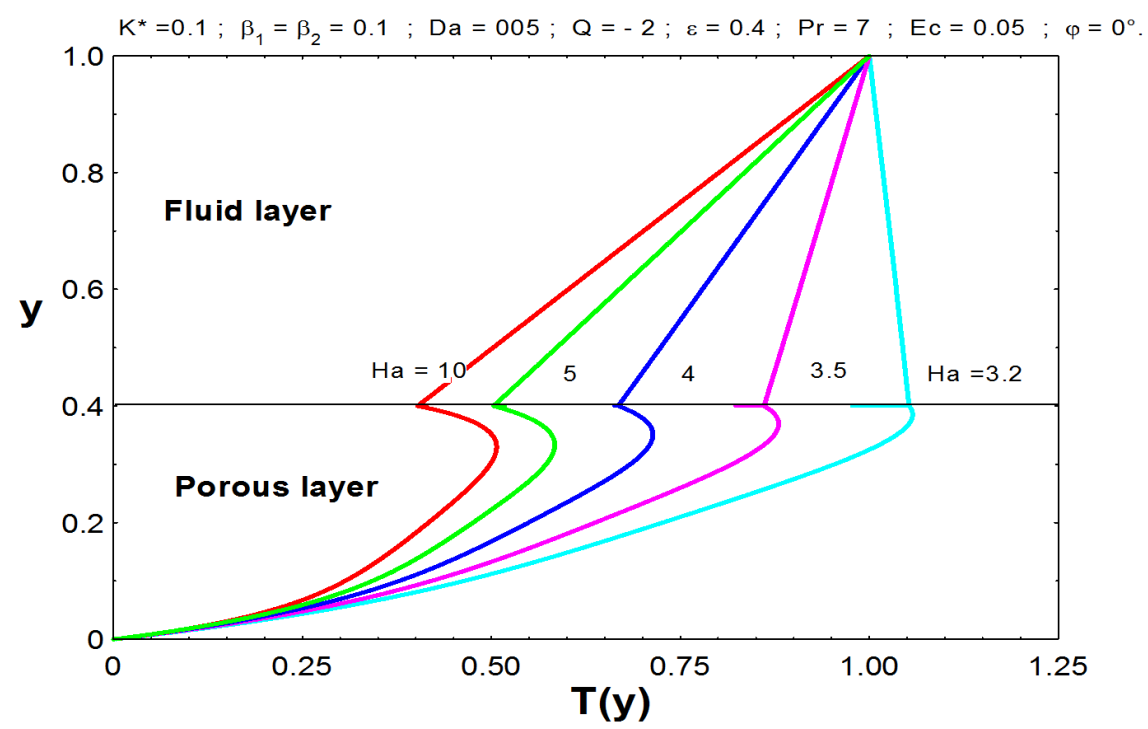

Figure 3. Horizontal temperature distribution for the entire system width.

\subsection{Velocity Profile, $u_{B}$, at the Interface}

Figure 4 show the effects of various values of the Hartmann number, $\mathrm{Ha}$, of varying the anisotropic ratio, $K^{\star}$, and the thickness ratio, $\mathcal{E}$, on the velocity profile $u_{B}$ at the interface when $\varphi=10^{\circ}, \beta_{1}=\beta_{2}=0.1, \operatorname{Pr}=7, Q=-3, E c=5 \times 10^{-3}, D a=$ $10^{-3}$. Because of the fact that $u_{B}$ is not physically defined at $\varepsilon=0$ (corresponding to the situation when the two layers are assimilated into a pure fluid layer) and at $\varepsilon=1$ (case for which the system would become a pure porous layer), all curves observed tend asymptotically to the pure fluid case when $\varepsilon \rightarrow 0$. As expected, in this limit, the effects of the anisotropic properties of the almost negligible porous layer are irrelevant. For intermediate values of the thickness ratio, it is seen that, as the porous layer becomes more and more important, the anisotropic effects of the porous medium become more significant (please refer [4]). It is also noticed that each curve for a given value of $K^{*}$ is distinguishable from others at a special point $\varepsilon_{c}$ that depend on the Hartmann number and below which the effects of anisotropy and magnetic field is irrelevant. For example, $\varepsilon_{c}=0.0744$ when $H a=1, \quad \varepsilon_{c}=0.0731$ when $H a=0.5$ and $\varepsilon_{c}=0.0722$ when $H a=0.005$. For each value of $K^{*}$, Figure 4 indicates that the velocity profile $u_{B}$ at the interface decreases as the Hartmann number increases.

\subsection{The Mean Heat Transfer Rate, $\mathbf{N} u_{\text {moy }}$}

The variations of Nusselt number with the Hartmann number and the anisotropic ratio is shown in Figure 5 as a function of the thickness ratio, $\mathcal{E}$, when $\mathrm{Da}$ $=75 \times 10^{-3}, \varphi=0^{\circ}, \operatorname{Pr}=7.0, E \mathcal{C}=0.05, \beta_{1}=\beta_{2}=0.001$, and $Q=50$. When the porous lining is negligible with respect to the fluid layer (i.e., when the thickness ratio is small), the heat transfer given by Equation (50), does not vary with the anisotropic ratio, $K^{\star}$. Then the Nusselt number decreases with the increase of the thickness ratio and the effect of anisotropy is predominant. Figure 5 indicates 


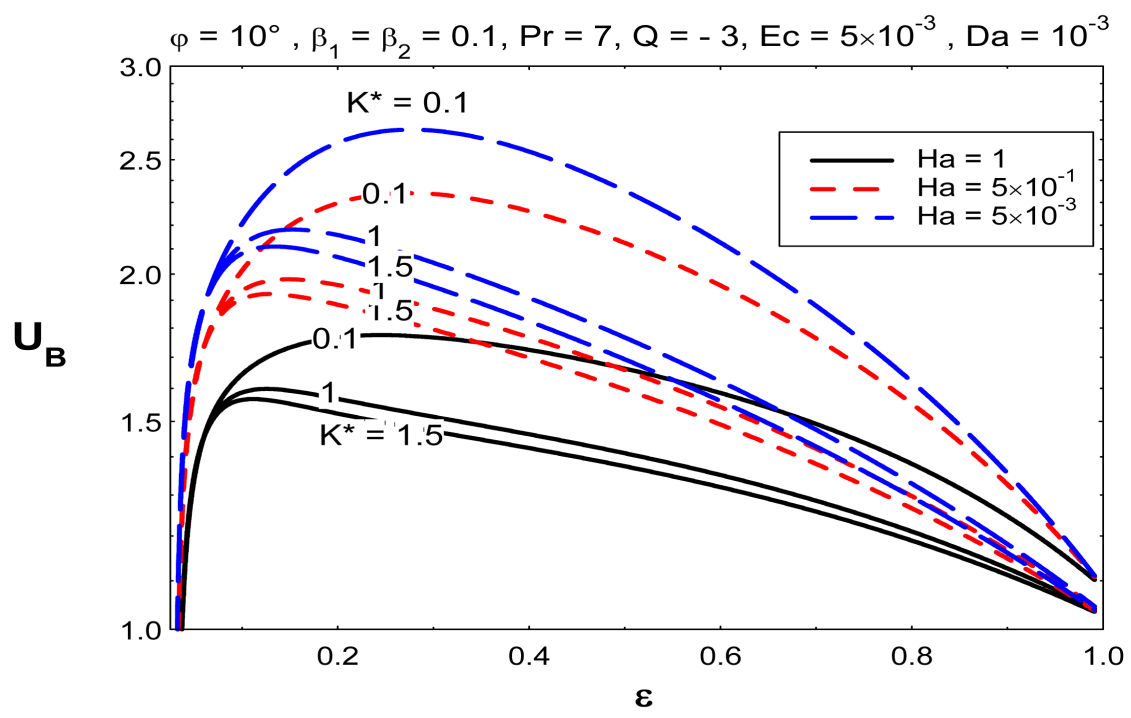

Figure 4. Velocity profile, $u_{B}$, at the interface.

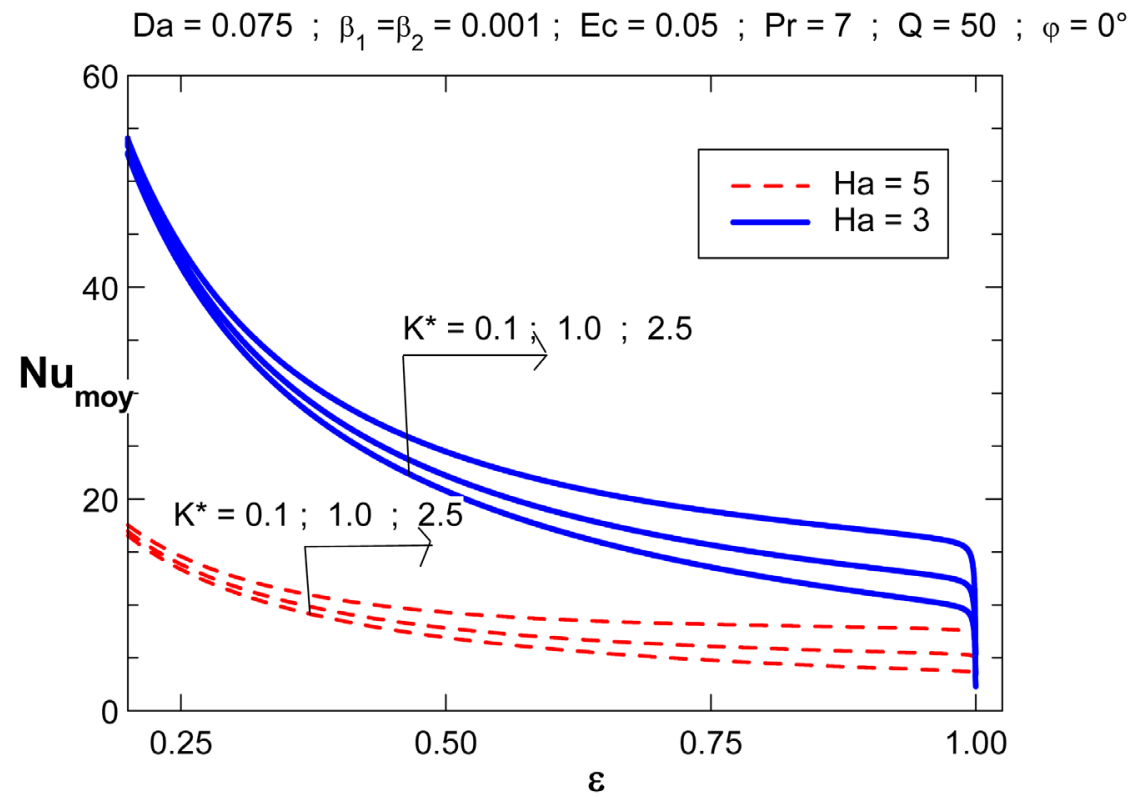

Figure 5. Effect of the Hartmann number on Nusselt number, $N u_{m o r}$

that the heat transfer through the entire system is enhanced when $K^{*}=2.5$ with respect to the isotropic case for which $K^{*}=1.0$. That is damaging to the aquatic species life. We also note that the increase of the Hartmann number corresponds to the decrease of the heat transfer when the porous layer becomes more and more important. What encourages the aquatic species life.

In Figure 6, we have plotted the average Nusselt number as a function of the thickness ratio, $\varepsilon$, with the variations of Hartmann number and the anisotropic orientation $\varphi$ for $D a=75 \times 10^{-3}, K^{*}=0.1, \operatorname{Pr}=7.0, E \mathcal{C}=0.05, \beta_{1}=\beta_{2}=0.001$, and $Q=50$. It is noticed that the Nusselt number have the same behavior as described previously, revealing that the heat transfer through the entire system for 


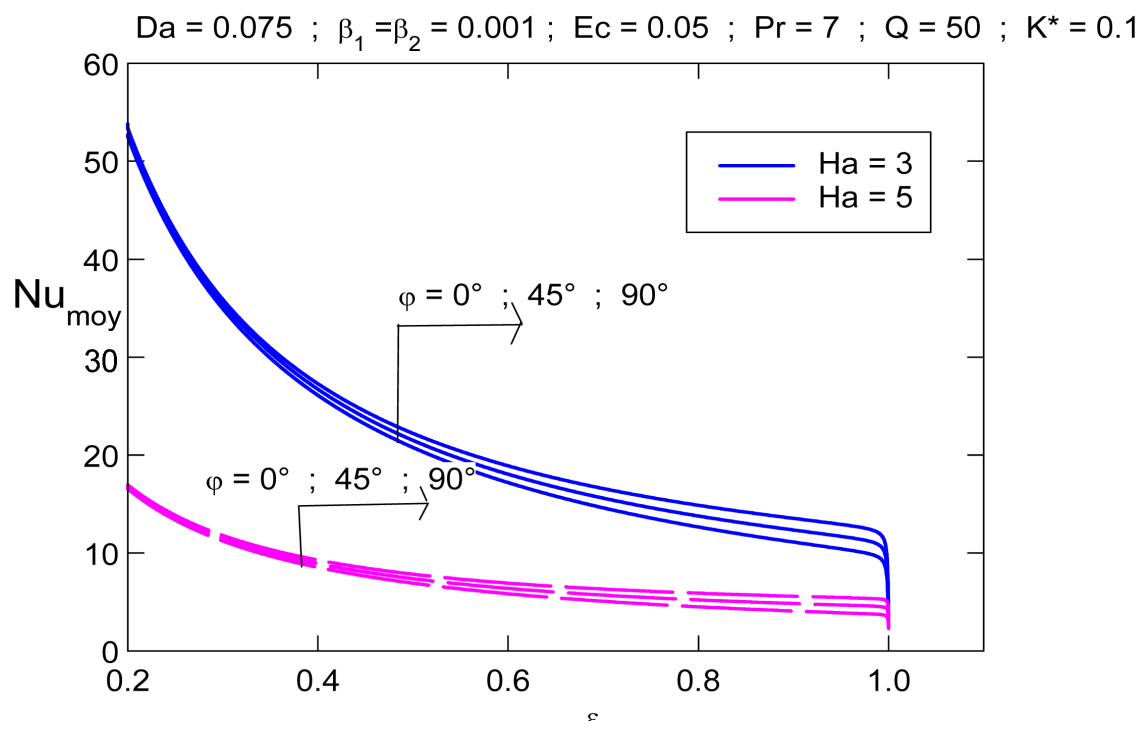

Figure 6. Effect of the Hartmann number and $\varphi$ on Nusselt number, $N u_{\text {mor }}$

a given value of Hartmann number is found to be maximum when $\varphi=90^{\circ}$ and minimum when $\varphi=0^{\circ}$.

The average Nusselt number is presented in Figure 7 as a function of the Darcy number, of the Hartmann number and of varying the anisotropic ratio when $\varphi=0^{\circ}, \operatorname{Pr}=7, E c=0.05, \beta_{1}=\beta_{2}=10^{-6}, \varepsilon=0.25$ and $Q=5$. From this figure it is clearly seen that the average Nusselt number decreases as the Hartmann number and the Darcy number increase, the highest decrease of the average Nusselt number occurs for the region of the low values of the Darcy number (i.e., in the porous zone where the effect of anisotropy is predominant). For high values of the Darcy number (case for which the system would become a pure fluid layer), no significant decrease occurs on the average Nusselt number which is not affected by the anisotropy ratio.

In Figure 8, the average Nusselt number is plotted as a function of the Darcy number for $K^{\star}=0.5, \operatorname{Pr}=7, E \mathcal{c}=0.05, \beta_{1}=\beta_{2}=10^{-6}, \varepsilon=0.25$ and $Q=5$ and various values of the Hartmann number and of $\varphi$. It is noticed that the average Nusselt number have the same behavior as described previously in Figure 7 and is found to be maximum when $\varphi=90^{\circ}$ and minimum when $\varphi=0^{\circ}$.

\section{Conclusion}

In this paper we have examined the effect of magnetic field on convection heat transfer through packed porous beds which consists of a horizontal fluid layer (river bed) and a porous zone with anisotropic permeability. The results obtained show that, the application of a magnetic field on the natural convection in the y-direction has some important changes on the structure of the convective flow and on convection heat transfer. It generates a reduction in the heat transfer and reduces considerably the velocity and temperature distribution. We have also examined the effect of anisotropic permeability ratio and anisotropic angle 


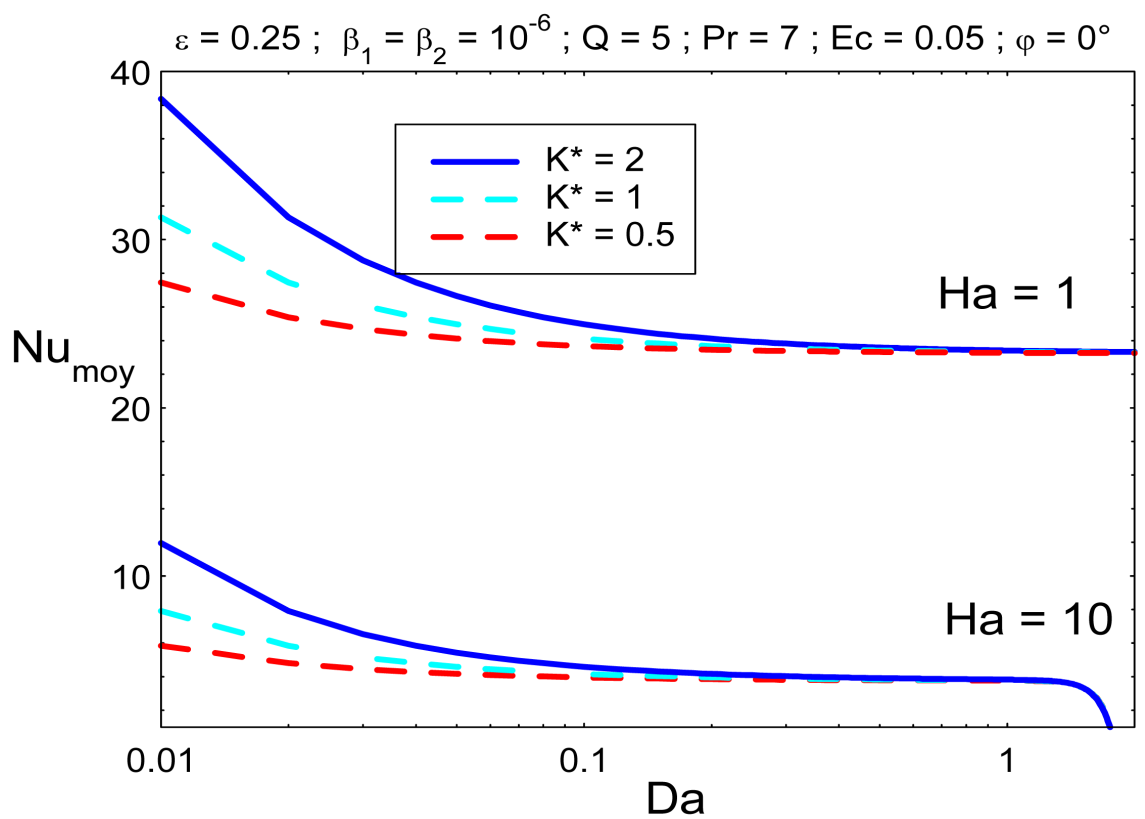

Figure 7. Effect of the Hartmann number and Da on Nusselt number, $N u_{\text {mor }}$.

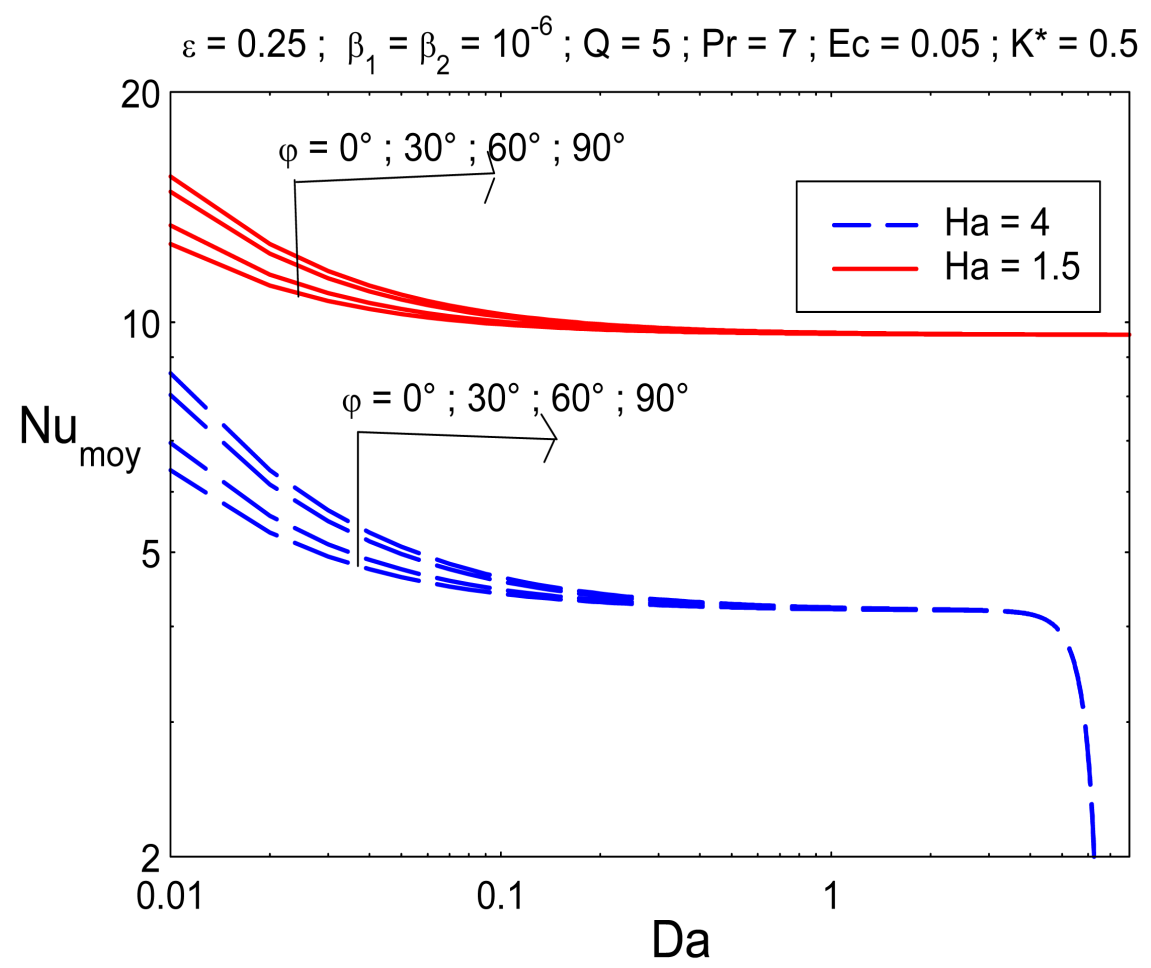

Figure 8. Effect of the Hartmann number and $\varphi$ on Nusselt number, $N u_{m o r}$

on the velocity profile, $u_{B}$, at the interface and on the average Nusselt number. The heat transfer, for a given value of the Hartmann number and for $\varphi=0^{\circ}$, is found to be maximum (minimum) when the permeability in the $y$-direction (x-direction) is bigger than the permeability in the $\mathrm{x}$-direction (y-direction) and for a given value of the anisotropic permeability ratio $\left(K^{*}=0.5\right)$, the average 
Nusselt number is found to be maximum when $\varphi=90^{\circ}$ and minimum when $\varphi=$ $0^{\circ}$.

\section{References}

[1] Ni, J., Beckerman, C. and Smith, T.F. (1993) Effect of an Electromagnetic Field on Natural Convection in Porous Medium. Fundam Heat Transf Electromagn Electrost Acoust Field ASME HTD, 248, 23-33.

[2] Alchaar, S., Vasseur, P. and Bilgen, E. (1995) Effects of a Magnetic Field on the Onset of Convection in a Porous Medium. Heat and Mass Transfer, 30, 259-267.

https://doi.org/10.1007/BF01602772

[3] Bian, W., Vasseur, P. and Bilgen, E. (1996) Effect of an External Magnetic Field on Buoyancy-Driven Flow in a Shallow Porous Cavity. Numerical Heat Transfer, Part $A$, 29, 625-638. https://doi.org/10.1080/10407789608913810

[4] Yovogan, J. and Degan, G. (2013) Effect of Anisotropic Permeability on Convective Heat Transfer through a Porous River Bed Underlying a Fluid Layer. Journal of Engineering Mathematics, 81, 127-140. https://doi.org/10.1007/s10665-012-9605-6

[5] Beavers, G.S. and Joseph, D.D. (1974) Boundary Conditions at a Naturally Permeable Wall. Journal of Heat Transfer, 96, 324-330.

[6] Farid, B., Benissad, S., Talbi, K. and Bouttout, A. (2007) Effet d'un champ magnétique externe sur la convection naturelle dans une enceinte rectangulaire tridimensionnelle. Jean-Jacques BEZIAN. JITH, Albi, France. ENSTIMAC, 5 p, <hal-00156640>.

[7] Banjer, H.M. and Abdullah, A.A. (2010) Convection in Superposed Fluid and Porous Layers in the Presence of a Vertical Magnetic Field. WSEAS Transactions on Fluid Mechanics, 5, 175-185.

[8] Garandet, J.P., Alboussiere, T. and Moreau, R. (1992) Buoyancy-Driven Convection in a Rectangular Enclosure with a Transverse Magnetic Field. International Journal of Heat and Mass Transfer, 35, 741-748. https://doi.org/10.1016/0017-9310(92)90242-K

[9] Degan, G., Zohoun, S. and Vasseur, P. (2002) Forced Convection in Horizontal Porous Channels with Hydrodynamic Anisotropy. International Journal of Heat and Mass Transfer, 45, 3181-3188. https://doi.org/10.1016/S0017-9310(02)00032-7

[10] Rudraiah, N. and Veerabhadraiah, R. (1977) Temperature Distribution in Couette Flow past a Permeable Bed. Proceedings of the Indian Academy of Science, Section $A, 86,537$.

[11] Rudraiah, N. and Veerabhadraiah, R. (1978) Effect of Buoyancy on the Free Surface Flow past a Permeable Bed. Wärme- und Stoffübertragung, 11, 265.

https://doi.org/10.1007/BF02587790 